\title{
Convergence analysis of hybrid cellular automata for topology optimization
}

\author{
C. L. Penninger $\cdot$ L. T. Watson $\cdot$ A. Tovar $\cdot$ J. E. Renaud
}

\begin{abstract}
The hybrid cellular automaton (HCA) algorithm was inspired by the structural adaptation of bones to their ever changing mechanical environment. This methodology has been shown to be an effective topology synthesis tool. In previous work, it has been observed that the convergence of the HCA methodology is affected by parameters of the algorithm. As a result, questions have been raised regarding the conditions by which HCA converges to an optimal design. The objective of this investigation is to examine the conditions that guarantee convergence to a Karush-Kuhn-Tucker (KKT) point. In this paper, it is shown that the HCA algorithm is a fixed point iterative scheme and the previously reported KKT optimality conditions are corrected. To demonstrate the convergence properties of the HCA algorithm, a simple cantilevered beam example is utilized. Plots of the spectral radius for projections of the design space are used to show regions of guaranteed convergence.
\end{abstract}

Keywords Fixed point iteration .

Spectral radius analysis .

Structural optimization.

Cellular automata convergence

\section{Introduction}

The hybrid cellular automaton (HCA) method is a biologically inspired algorithm capable of topology synthesis that was developed to simulate the behavior of bone remodeling as presented by Tovar (2004). This methodology is termed a hybrid technique because it couples global information obtained from the finite element (FE) method with the local relationships utilized by cellular automata (CA) computing. The incorporation of global information increases the efficiency of the HCA algorithm. While KarushKuhn-Tucker (KKT) optimality conditions have been derived for the HCA algorithm (Tovar et al., 2006), some questions have been raised whether HCA actually converges to an optimal structure. It is known that it is possible to obtain different converged HCA solutions for the same application and these solutions cannot all be optimal. An application of CA to truss design has been rigorously shown to converge to an optimal solution (Slotta et al., 2002). Following from this work, an analysis of the convergence of HCA as a fixed point iteration is conducted to determine the conditions under which this algorithm converges to a KKT point.

The concept of CA was pioneered by John von Neumann in the late 1940s. Von Neumann, who first proposed the concept of a stored program for the digital computer, envisioned a system with the capability of solving very complex problems. He imagined a machine with the complexity of the human brain that also contained self-control and self-repair mechanisms. Such a device would have the capability of repairing itself with available material. As a result, von Neumann developed the concept of a fully discrete universe made up of lattice cells that behave as automata. Each cell would evolve simultaneously in discrete time steps according to a simple rule involving its own state and the states of neighboring cells. According to Burks (1970), the first self-replicating CA proposed by von Neumann was composed of a two-dimensional square lattice composed of several thousand cells. Each cell had the possibility of up to 29 states. The local rule involved the state of each cell and its four nearest neighbors, located north, south, east, and west. This CA model was so complex that it has only been partially implemented on a computer (Pesavento, 1995). The von Neumann rule has the property of universal computation, meaning there exists an initial configuration of the $\mathrm{CA}$ that results in any finite algorithmic computation (i.e., the $\mathrm{CA}$ implements a universal Turing machine). This implies that any 
computer program can be simulated by an automaton (Chopard and Droz, 1998). Thus, complex and unexpected behavior can emerge from CA rules. CA are a model of massively parallel computation (Chopard and Droz, 1998). CA were brought to the attention of a wide audience in 1970, when John Conway proposed his now famous Game of Life (Gardner, 1970). CA are currently being used to study artificial life in an attempt to better understand real life and the behavior of living species through computer models.

A cellular automaton is a discrete dynamical system that has been utilized to study idealizations of complex physical processes. The basis for CA is that an overall global behavior can emerge from local rules acting over an automaton that possesses only local state information. A cellular automaton (CA) consists of a regular lattice of cells, each described by a finite dimensional vector of states. The lattice can exist in any finite number of dimensions. CA evolve in discrete time; therefore, the state of a cell at a discrete time depends on a local rule that utilizes information from a finite number of nearby cells, known as a neighborhood. Both the local rules and neighborhoods are consistent for each site of the lattice and are applied for all generations in time.

As previously mentioned, CA are a massively scalable approach that lends itself to a variety of disciplines. For instance, CA have been applied to shape and topology optimization (Kita and Toyoda, 2000), the design of fiber reinforced composites (Setoodeh et al., 2006a), wave propagation in excitable media (Weimar et al., 1992a,b), and for the solution of heat transfer problems (Lowekamp et al., 1996). CA rules have recently been devised for the simultaneous analysis and design of simple two-dimensional structures (Gürdal and Tatting, 2000; Tatting and Gürdal, 2000). While the CA approach works well for many problems, the size and complexity of a problem is often limited by inefficient parallelization on massively parallel processors (MPPs) (Gürdal and Watson, 2001). Various techniques have been developed to improve the efficiency of the CA parallelization, such as block synchronous and block pipeline parallel implementations (Setoodeh et al., $2006 \mathrm{~b})$. Other related work on $\mathrm{CA}$ can be found in Hajela and Kim (2001), Abdalla and Gürdal (2004), Abdalla et al. (2005), Canyurt and Hajela (2005), Missoum et al. (2005), Setoodeh et al. (2005), and Ryoo et al. (2007).

In Tovar et al. (2006) the HCA methodology for topology optimization was developed. This approach reduces numerical instabilities by using CA principles, as opposed to filtering techniques. In conventional
CA methods, a global analysis of field states is not performed. As previously mentioned, the HCA method was originally developed for the simulation of the bone remodeling process. This model assumes that bone remains locally isotropic and structural changes are driven by an applied mechanical stimulus. Therefore, it is assumed that the distribution of material in a region of bone is altered to uniformly distribute the effects of the applied stimulus. The HCA method has many similarities to topology optimization techniques, although not being a formal optimization method itself. This framework has been shown to not only be effective at generating bone architecture, but it also has been extended to the areas of compliant mechanism synthesis and crashworthiness design (Patel, 2007).

\section{Optimality of HCA Solutions}

The basis for the HCA method is that the CA lattice is used to represent the connected cellular network of osteocytes in bones. Relying on the assumption that osteocytes sense the local mechanical stimulus experienced in bone, each cell of the $\mathrm{CA}$ lattice contains an osteocyte or a number of osteocytes, surrounded by mineralized tissue. This model assumes that bone remains locally isotropic and changes in relative density are driven by a mechanical stimulus target differential. Therefore, the state of cell $i$ is defined by the relative (or normalized) density $x_{i}(t)$, the mechanical stimulus $S_{i}(t)$, and the error signal $e_{i}(t)$, i.e.,

$\psi_{i}(t)=\left(\begin{array}{c}x_{i}(t) \\ S_{i}(t) \\ e_{i}(t)\end{array}\right)$.

Since the relative density of each cell can vary throughout the remodeling process, changes in this parameter will result in a change in the modulus of the material. Thus, the modulus at a location $i$ is calculated using a power law relationship,

$E_{i}(t)=E_{0 i} x_{i}(t)^{p}$,

where $E_{0 i}$ is the base modulus for each cell, typically set to the modulus of fully dense bone (assumed to be equivalent to cortical bone), and $p$ is an empirical value, typically satisfying $2 \leq p \leq 3$ (Currey, 1988; Bendsøe, 1989; Rozvany and Zhou, 1991).

It is assumed that bone adapts to its environment to obtain a state of equilibrium, corresponding to a local mechanical stimulus target. Therefore, the strength of the remodeling signal is measured by the error between the effective stimulus sensed and the stimulus target,

$e_{i}(t)=\bar{S}_{i}(t)-S_{i}^{*}$, 


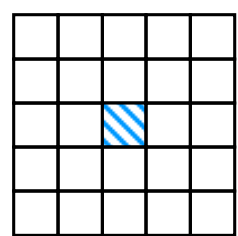

(a) Empty $(\hat{N}=0)$

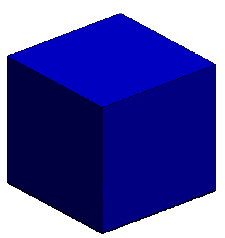

(e) $\operatorname{Empty}(\hat{N}=0)$

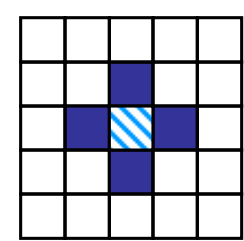

(b) Von Neumann $(\hat{N}=4)$

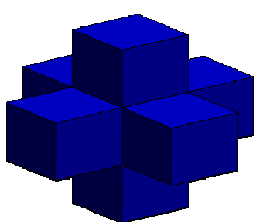

(f) Von Neumann $(\hat{N}=6)$

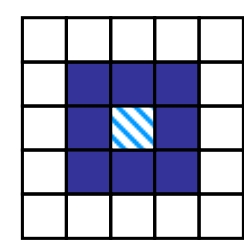

(c) $\operatorname{Moore}(\hat{N}=8)$

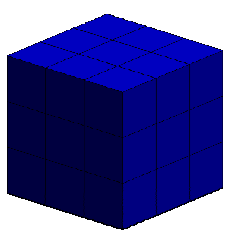

(g) Moore $(\hat{N}=26)$

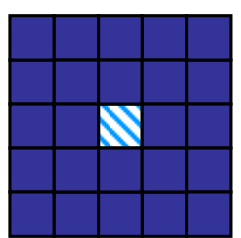

(d) Extended $(\hat{N}=24)$

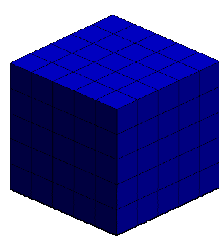

(h) Extended $(\hat{N}=124)$

Fig. 1 Common 2D ((a)-(d)) and 3D ((e)-(h)) CA neighborhoods. $\hat{N}$ depicts the number of neighboring cells.

where $\bar{S}_{i}(t)$ is the effective mechanical stimulus, which incorporates information from neighboring cells, and $S_{i}^{*}$ is the stimulus target. The stimulus target for bone remodeling studies is defined from clinical observations. Local rules $\mathbf{R}$ are then used to update the material distribution in the domain, based on the magnitude of the stimulus error signal.

The set of local rules $\mathbf{R}$ that govern the evolution of the state of each cell of the automaton operate according to information gathered from the cells in a prescribed neighborhood. In general, there is no restriction placed on the size of a neighborhood, only that the neighborhoods are consistent for each cell. The use of a neighborhood is similar to filtering techniques used in topology optimization to avoid numerical instabilities such as mesh dependency and checkerboarding.

As mentioned before, it is theorized that mechanical signals are detected in bone by osteocytes, which are distributed throughout the bone matrix. These osteocytes extend cellular processes in order to transmit signals to their neighbors, forming a highly interconnected cellular network. Therefore, in the context of bone remodeling, local information gathered from neighboring cells can be viewed as the communication via the osteocytic network. In the original implementation of HCA, the effective mechanical stimulus sensed by each cell $\bar{S}_{i}(t)$ is expressed as the average of the stimuli sensed by all of the cells within the designated neighborhood,

$\bar{S}_{i}(t)=\frac{S_{i}(t)+\sum_{k \in N(i)} S_{k}(t)}{\hat{N}(i)+1}$,

where $S_{i}(t)$ is the state of mechanical stimulus at location $i, S_{k}(t)$ represents the mechanical stimulus sensed by the $k^{\text {th }}$ neighbor, $N(i)=$ indices of neighbors of cell $i\}$, and $\hat{N}(i)=|N(i)|$ is the total number of neighbors in the neighborhood. In practice, the size of the neighborhood is typically restricted to the adjacent cells but it can also be extended. Some common two- and three-dimensional neighborhood layouts are displayed in Fig. 1.

The local rules $\mathbf{R}$ utilized by the HCA framework are designed to model the process of bone functional adaptation to variations in its mechanical environment. These rules $R_{i}$ control the remodeling activities, i.e., formation and resorption in the bone. The local rules are formulated to operate on the error signal $e_{i}(t)$ that incorporates information from each cell of the CA and its neighborhood to determine whether material should be added or removed. These rules drive the evolution of the material distribution, which is the outcome of the remodeling process. In Tovar's work, control based rules and a ratio based rule, following the principles of fully stressed design, are presented (Tovar et al., 2007). This work will only focus on the proportional, integral, and derivative (PID) control strategy.

The HCA method employs a PID control strategy whose input is the mechanical stimulus measured for each cell and its neighbors. The purpose of this rule is to drive the effective state of each cell to the mechanical stimulus target $S^{*}$. The reasoning behind proportional control is that it is assumed that osteoclastic or osteoblastic activity occurs in proportion to the error between a local effective mechanical stimulus and the stimulus target. Various computational models of bone remodeling use some form of proportional control in their remodeling rule (Fyhrie and Carter, 1986; Huiskes et al., 1987; Huiskes et al., 2000; Ruimerman et al., 2005). Integral control provides a pathway for including a sense of memory 
in the adaptation process. Consequently, this can be interpreted as osteocytes storing information from previous states, i.e., $e_{i}(t-1), \ldots, e_{i}(t-T)$. Thus, the remodeling activity is proportional to the cumulative error. The rate of osteoblastic and osteoclastic activity depending on a prediction of the future error signal, based on the current and previous error signals $e_{i}(t)$ and $e_{i}(t-1)$, represents a form of derivative control. Hence, the change in relative density $\Delta x_{i}(t)$ for a cell at location $i$ is given as

$\Delta x_{i}(t)=c_{p} e_{i}(t)+c_{i} \int_{0}^{t} e_{i}(\tau) d \tau+c_{d} \Delta e_{i}(t)$,

where $c_{p}, c_{i}$, and $c_{d}$ are the proportional, integral, and derivative control gains, respectively. Therefore, the material update for each cell is

$$
\begin{aligned}
x_{i}(t+1)= & \min \left\{\operatorname { m a x } \left\{0, x_{i}(t)+c_{p} e_{i}(t)\right.\right. \\
& \left.\left.+c_{i} \int_{0}^{t} e_{i}(\tau) d \tau+c_{d} \Delta e_{i}(t)\right\}, 1\right\} .
\end{aligned}
$$

It has been observed that this PID control strategy reduces numerical instabilities and improves convergence performance of the HCA algorithm.

Recall that this analysis began with the assumption of a stimulus equilibrium that was motivated by the physiology and occurs when the stimulus error in (3) goes to zero. This represents a criterion for each cell to enforce. One could reasonably ask what mathematical problem this criterion is the solution to. The answer turns out to be the multiobjective optimization problem given below. It has been shown that the HCA framework is capable of topology synthesis, although not being a formal topology optimization method itself (Tovar et al., 2006). Previously, Tovar el al. (2007) derived a new set of design rules for the HCA methodology for structural topology optimization, based on the Karush-Kuhn-Tucker (KKT) optimality conditions. This formulation is based on a multiobjective optimization problem for minimizing both mass and strain energy written as

$\min _{0 \leq x \leq 1} f(U)+g(M)$,

where $f(U)$ is a function of the strain energy $U, g(M)$ is a function of the mass $M$, and $x$ is the vector of relative densities for each cell. The Lagrangian of this optimization problem can be written as

$L=f(U)+g(M)+\left(\lambda^{1}\right)^{T}(x-1)-\left(\lambda^{0}\right)^{T} x$,

where $\lambda^{0}$ and $\lambda^{1}$ are the Lagrange multiplier vectors associated with the inequality constraints. The
KKT necessary conditions for the multiobjective optimization problem are given by

$$
\begin{aligned}
& \frac{\partial L}{\partial x_{i}}=\frac{\partial f(U)}{\partial U} \frac{\partial U}{\partial x_{i}}+\frac{\partial g(M)}{\partial M} \frac{\partial M}{\partial x_{i}}+\lambda_{i}^{1}-\lambda_{i}^{0}=0, \\
& \lambda_{i}^{1} \geq 0 \\
& \lambda_{i}^{0} \geq 0 \\
& \lambda_{i}^{1}\left(x_{i}-1\right)=0 \\
& \text { and } \\
& \lambda_{i}^{0} x_{i}=0 .
\end{aligned}
$$

For an interior point, i.e., $0<x_{i}<1$, the Lagrange multipliers $\lambda_{i}^{1}=\lambda_{i}^{0}=0$, satisfying (10)-(13). Therefore, the optimality conditions are satisfied if

$\frac{\partial f(U)}{\partial U} \frac{\partial U}{\partial x_{i}}+\frac{\partial g(M)}{\partial M} \frac{\partial M}{\partial x_{i}}=0$.

The strain energy $U$ can be approximated using the FE method to yield the equilibrium equations

$K u=f$,

where $K$ is the global stiffness matrix, $u$ is the global nodal displacement vector, and $f$ is the global force vector. Thus, $U$ can be written as

$U=\frac{1}{2} u^{T} K u=\frac{1}{2} \sum_{i=1}^{N} u_{i}^{T} k_{i} u_{i}$

where $k_{i}$ is the element stiffness matrix, $u_{i}$ is the element nodal displacement vector, and $N$ is the total number of elements. Using (16), $\partial U / \partial x_{i}$ can be expressed as

$\frac{\partial U}{\partial x_{i}}=\frac{1}{2}\left(\frac{\partial u^{T}}{\partial x_{i}} K u+u^{T} \frac{\partial(K u)}{\partial x_{i}}\right)$,

which can be reduced to

$\frac{\partial U}{\partial x_{i}}=-\frac{1}{2} u^{T} \frac{\partial K}{\partial x_{i}} u$.

Since $x_{i}$ is only present in its corresponding element stiffness matrix $k_{i}$ and, similarly, the only active part of the global nodal displacement vector is the element nodal displacement vector $u_{i}$, (18) can be rewritten on an elemental basis

$\frac{\partial U}{\partial x_{i}}=-\frac{1}{2} u_{i}^{T} \frac{\partial k_{i}}{\partial x_{i}} u_{i}$

Assuming that each element in the FE model is comprised of isotropic elastic material, the stiffness matrix for each element can be written as

$k_{i}=k_{0 i} x_{i}^{p}$,

where $k_{0 i}$ corresponds to the elastic stiffness matrix when $E_{i}=E_{0 i}$ (refer to (2)). Thus, substituting (20) into (19) yields

$\frac{\partial U}{\partial x_{i}}=-\frac{1}{2} u_{i}^{T} \frac{\partial\left(k_{0 i} x_{i}^{p}\right)}{\partial x_{i}} u_{i}=-\frac{1}{2} u_{i}^{T} p k_{0 i} x_{i}^{p-1} u_{i}$. 
The strain energy density $U_{i}$ at a discrete location $i$ can be expressed as

$U_{i}=\frac{1}{2 v_{0 i}} u_{i}^{T} k_{0 i} x_{i}^{p} u_{i}$,

where $v_{0 i}$ is the constant volume of each element. Inserting $U_{i}$ into (21) yields

$\frac{\partial U}{\partial x_{i}}=-p v_{0 i} \frac{U_{i}}{x_{i}}$.

An expression for $\partial M / \partial x_{i}$ can be derived from the total mass $M$, which can be written as

$M=\sum_{i=1}^{N} m_{i}=\sum_{i=1}^{N} x_{i} m_{0 i}$,

where $m_{i}$ is the mass of an element and $m_{0 i}$ is the maximum mass of the element. Therefore, $\partial M / \partial x_{i}$ is expressed as

$\frac{\partial M}{\partial x_{i}}=m_{0 i}$.

By substituting (23) and (25) into (14) and rearranging terms, the optimality condition for an interior point can be rewritten as

$\frac{p v_{0 i}}{m_{0 i}} \frac{U_{i}}{x_{i}}=\frac{\partial g(M) / \partial M}{\partial f(U) / \partial U}$.

For the case where $f(U)=\omega_{1} U$ and $g(M)=\omega_{2} M$, $\partial f(U) / \partial U=\omega_{1}$ and $\partial g(M) / \partial M=\omega_{2}$. Therefore, for an interior point the optimality condition from (26) yields

$\frac{p}{\rho_{0 i}} \frac{U_{i}}{x_{i}}=\frac{\omega_{2}}{\omega_{1}}$,

where $\rho_{0 i}$ is the density of the solid element, written as

$\rho_{0 i}=\frac{m_{0 i}}{v_{0 i}}$.

Thus, from this optimality condition, the mechanical stimulus $S_{i}$ and equilibrium stimulus $S_{i}^{*}$ can be represented as

$S_{i}=\frac{U_{i}}{x_{i}}$

and

$S_{i}^{*}=\frac{\omega_{2}}{\omega_{1}} \frac{\rho_{0 i}}{p}$.

If the relative density $x_{i}$ of an element is saturated, i.e., $x_{i}=0$ or $x_{i}=1$, the optimality condition given by (27) is no longer valid. For the case of $x_{i}=0$, $\lambda_{i}^{1}=0$ must also be true in order to satisfy the condition of (12). Therefore, the conditions in (10),
(12), and (13) are satisfied. Combining the remaining optimality conditions from (9) and (11) yields

$\lambda_{i}^{0}=\left.\frac{\partial f(U)}{\partial U} \frac{\partial U}{\partial x_{i}}\right|_{x_{i}=0}+\left.\frac{\partial g(M)}{\partial M} \frac{\partial M}{\partial x_{i}}\right|_{x_{i}=0} \geq 0$.

Utilizing the assumption that $f(U)=\omega_{1} U$ and $g(M)=\omega_{2} M$ and substituting $\partial U /\left.\partial x_{i}\right|_{x_{i}=0}=0$ from (21) and $\partial M /\left.\partial x_{i}\right|_{x_{i}=0}=m_{0 i}$ from (25) yields the optimality condition

$\lambda_{i}^{0}=\omega_{2} m_{0 i} \geq 0$.

This condition implies that $0=S_{i} \leq S_{i}^{*}$ if $x_{i}=0$.

In the case that $x_{i}=1, \lambda_{i}^{0}=0$ must also be true in order to satisfy the condition of (13). Therefore, the conditions in (11), (12), and (13) are satisfied. Combining the remaining optimality conditions from (9) and (10) yields

$\lambda_{i}^{1}=-\left.\frac{\partial f(U)}{\partial U} \frac{\partial U}{\partial x_{i}}\right|_{x_{i}=1}-\left.\frac{\partial g(M)}{\partial M} \frac{\partial M}{\partial x_{i}}\right|_{x_{i}=1} \geq 0$.

Again, utilizing the assumption that $f(U)=\omega_{1} U$ and $g(M)=\omega_{2} M$ and substituting

$\partial U /\left.\partial x_{i}\right|_{x_{i}=1}=-p v_{0 i} U_{i} /\left.x_{i}\right|_{x_{i}=1}$

from (23) and $\partial M /\left.\partial x_{i}\right|_{x_{i}=1}=m_{0 i}$ from (25) yields the optimality condition

$\lambda_{i}^{1}=\left.\omega_{1} p v_{0 i} \frac{U_{i}}{x_{i}}\right|_{x_{i}=1}-\omega_{2} m_{0 i} \geq 0$,

which can be written as

$\left.\frac{U_{i}}{x_{i}}\right|_{x_{i}=1} \geq \frac{\omega_{2}}{\omega_{1}} \frac{\rho_{0 i}}{p}$.

This condition implies that $\left.S_{i}\right|_{x_{i}=1} \geq S_{i}^{*}$.

\section{Analysis of Convergence}

This paper considers a particular HCA of the form

$x_{i}(t+1)=\min \left\{\max \left\{0, x_{i}(t)+c_{p} e_{i}(t)\right\}, 1\right\}$.

It will be shown that the HCA iteration can be written in the form of a fixed point iteration

$x(t+1)=G(x(t))$,

where $G(x(t))$ is the function representing the HCA update. In mathematics, a fixed point $\bar{x}$ of a function $G(x)$ is a point that is mapped to itself by the function: $G(\bar{x})=\bar{x}$ (Isaacson and Keller, 1966). There are numerous theorems in different parts of mathematics that guarantee that these functions, if they satisfy certain conditions, have at least one fixed point. The necessary conditions for the function representing the HCA update $G(x(t))$ to converge to a fixed point will be discussed here. 
In order to show that the HCA update can be written in the form of a fixed point iteration, the mechanical stimulus error signal $e_{i}(t)$ must be written in terms of $x_{i}(t)$. From (3), the mechanical stimulus error signal is represented by

$e_{i}(t)=\bar{S}_{i}(t)-S_{i}^{*}$.

Recall that the effective stimulus $\bar{S}_{i}(t)$ is the average mechanical stimulus over the desired neighborhood. For simplicity, this work will consider an empty neighborhood, i.e., $\hat{N}=0$, which implies $\bar{S}_{i}(t)=$ $S_{i}(t)$, consistent with the optimality condition $S_{i}=S_{i}^{*}$ from (27). In fact, Tovar's HCA converging to $e_{i}=\bar{S}_{i}-S_{i}^{*}=0$ with $\bar{S}_{i} \neq S_{i}$ does not satisfy even his own optimality criterion (Tovar et al., 2006). The mechanical stimulus $S_{i}(t)$ was determined from the derivation of the optimality conditions for the multiobjective optimization above (29). Thus, inserting this expression into (38) yields

$e_{i}(t)=\frac{U_{i}(t)}{x_{i}(t)}-S_{i}^{*}$.

Utilizing the definition of strain energy density $U_{i}(t)$ from (22) and simplifying the result yields

$e_{i}(t)=\frac{1}{2 v_{0 i}} u_{i}^{T}(t) k_{0 i} x_{i}^{p-1}(t) u_{i}(t)-S_{i}^{*}$.

The elemental displacement vector $u_{i}^{T}(t)$ can be determined from the equilibrium equation for an element

$u_{i}(t)=\left(K^{-1}(x(t)) f\right)_{I_{i}}$,

where $I_{i}=$ indices of nodal displacements of element $i$ \}. Therefore, (40) can be written as

$$
\begin{aligned}
e_{i}(t)= & \frac{1}{2 v_{0 i}}\left(K^{-1}(x(t)) f\right)_{I_{i}}^{T} k_{0 i} x_{i}^{p-1}(t) \\
& \times\left(K^{-1}(x(t)) f\right)_{I_{i}}-S_{i}^{*} .
\end{aligned}
$$

Substituting this expression into the HCA update for a single cell yields

$$
\begin{aligned}
x_{i}(t+1)= & \min \left\{\operatorname { m a x } \left\{0, x_{i}(t)+c_{p} \frac{x_{i}^{p-1}(t)}{2 v_{0 i}}\right.\right. \\
& \times\left(K^{-1}(x(t)) f\right)_{I_{i}}^{T} k_{0 i} \\
& \left.\left.\times\left(K^{-1}(x(t)) f\right)_{I_{i}}-S_{i}^{*}\right\}, 1\right\} .
\end{aligned}
$$

Therefore, since the HCA update for each cell can be written in terms of $x(t)$, it follows that the HCA update for the entire CA lattice can be written as

$x(t+1)=G(x(t))$.

Thus, it is shown that the HCA update is fixed point iteration.
If the HCA iteration is converging to a fixed point $\bar{x}$, then it must be true that

$\|x(t+1)-\bar{x}\| \rightarrow 0$.

Using (37), the definition of a fixed point, and the Mean Value Theorem gives

$$
\begin{aligned}
\|x(t+1)-\bar{x}\| & =\|G(x(t))-G(\bar{x})\| \\
& =\|D G(\hat{x})(x(t)-\bar{x})\| \\
& \leq\|D G(\hat{x})\| \|(x(t))-\bar{x}) \|
\end{aligned}
$$

for any vector norm $\|\cdot\|$ and for some $\hat{x}$ between $\bar{x}$ and $x(t)$. This holds for $0<x_{i}(t)<1$, and also at the boundary values if $x_{i}(t)=\bar{x}_{i}=0$ or $x_{i}(t)=\bar{x}_{i}=1$.

If the operator norm

$\|D G(\hat{x})\| \leq L<1$

for some constant $L$, vector norm $\|\cdot\|$, and every $\hat{x}$, then $G(x)$ has a unique fixed point $\bar{x}$ and the HCA iteration converges to $\bar{x}$ for any starting point $0<x_{i}(0)<1$ (this is the contraction mapping theorem). For each $\epsilon>0$ there exists a vector norm $\|\cdot\|$ such that

$\rho(D G(\bar{x})) \leq\|D G(\bar{x})\|<\rho(D G(\bar{x}))+\epsilon$,

where

$\rho(D G(\bar{x}))=\max _{1 \leq j \leq s}\left|\lambda_{j}\right|$

$\left(\lambda_{1}, \ldots, \lambda_{s}\right.$ are the eigenvalues of $\left.D G(\bar{x})\right)$

is the spectral radius of the Jacobian matrix $D G(\bar{x})$, and (48) holds for all $x$ in a neighborhood of $\bar{x}$ (Isaacson and Keller, 1966). Hence if

$\rho(D G(\bar{x}))<1$,

then the HCA iteration converges to the fixed point $\bar{x}$ for any initial $x(0)$ sufficiently close to $\bar{x}$. Note that if $D G(x)$ is symmetric, then $\|D G(x)\|_{2}=\rho(D G(x))$, and so $\rho(D G(x))<1$ on $0 \leq x \leq 1$ would guarantee HCA converges for any $0 \leq x(0) \leq 1$. For nonsymmetric $D G(x)$, the norm in (48) changes with $x$, so HCA convergence is guaranteed only in a neighborhood of $\bar{x}$.

\section{Numerical Example}

To analyze the convergence of HCA to an optimal point, a cantilevered structure modeled with four cells is considered (Fig. 2). For this example, a one to one mapping is used between the cells of the CA lattice and the FE model. Four-noded rectangular finite elements are used. The nodes along the left edge of the cantilever are constrained in both coordinate directions to have zero applied displacements so that the nodes are fixed. A downward force of $1 \mathrm{~N}$ is applied to the node in the bottom right corner of the lattice. For simplicity, the dimensions of the structure are $1 \mathrm{~m}$ in width by $1 \mathrm{~m}$ in height. As 
mentioned before, the HCA algorithm operates on the relative density of each cell, therefore, the density is interpolated between zero and one, using a power law relationship (2) with exponent $p=3$. The Young's modulus was set at $E=1 \mathrm{~Pa}$ and the Poisson's ratio to $\nu=0.3$. The equilibrium stimulus was set at $S_{j}^{*}=1.2 \mathrm{~Pa}$ for $j=1,2,3,4$, i.e., each cell in the lattice is seeking the same target stimulus. For this equilibrium stimulus, it can be shown using (30) that the corresponding objective function weights in (7) are $\omega_{1}=0.2174$ and $\omega_{2}=0.7826$. The CA implementation used for the following numerical experiments is given precisely by (43), and averaging is not done for the reasons previously given.

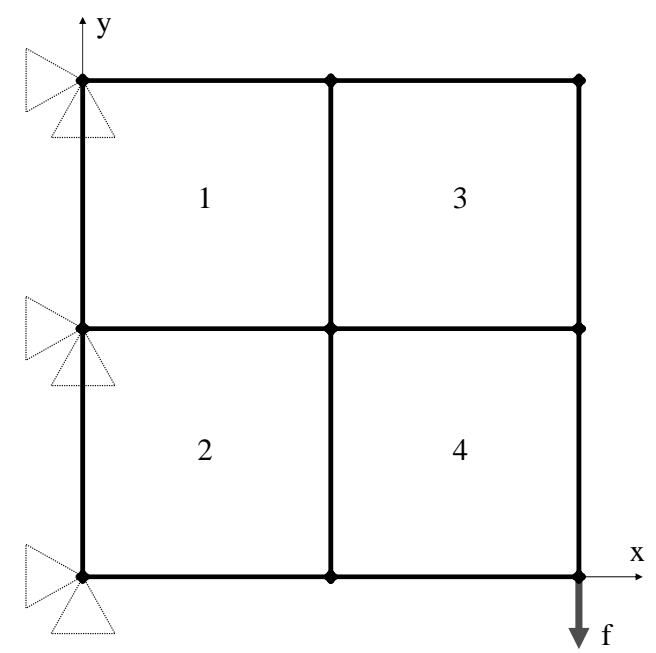

Fig. 2 Simple $2 \times 2$ cantilevered structure.

The optimal density distribution for this example was found to be $x=(0.999,0.999,0.001,0.999)$, as displayed in Fig. 3. Convergence was defined as no change in the objective function (7) or the design variable values $x$ larger than $10^{-7}$. It is shown that Cell 3 seeks the minimum density, while Cells 1, 2, and 4 seek a fully dense state. This result was verified with MATLAB's FMINCON (a sequential quadratic programming algorithm).

Since the HCA algorithm is a fixed point iterative approach, it is possible that there are multiple fixed points of which only one is a physical optimum point. The path that the HCA algorithm takes to a fixed point is affected by a number of parameters, for example, the material properties, material parameterization, the initial density distribution $x(0)$, and the proportional constant $c_{p}$. The spectral radius was evaluated for arbitrary starting values for combinations of two cells (i.e., Cells 1 and 2, Cells 1 and 3, etc.) while the remaining two cells were maintained at full density, for $c_{p}=0.05$ (Fig. 4). These plots display the spectral radius over projections of the design space.

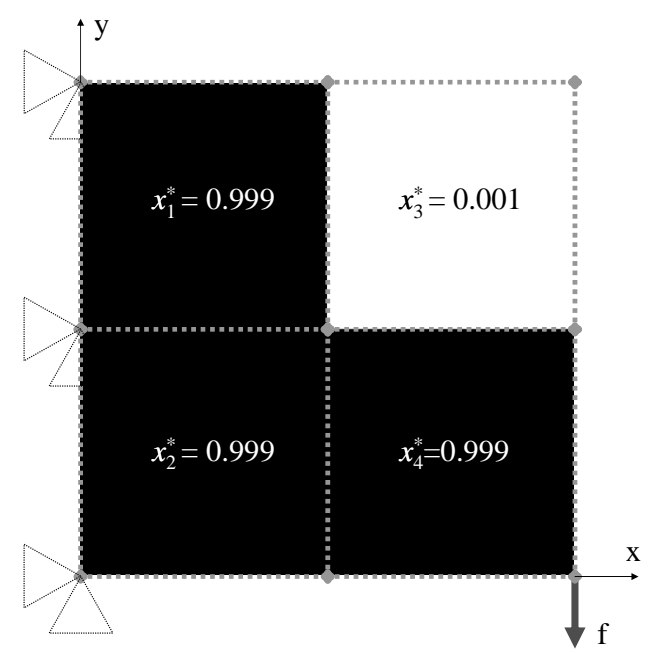

Fig. 3 Optimal density distribution for cantilevered structure.

Unexpectedly, it was found that there are disconnected regions in which the spectral radius is less than one. Therefore, if the initial density distribution falls within a region of spectral radius less than one that does not contain the optimal solution, convergence to an optimal solution is not guaranteed. In fact, for this problem it was observed that local minimum points (fixed points) may reside in these disconnected regions of spectral radius less than one.

The convergence properties of the HCA method are also affected by the value of $c_{p}$. The spectral radius was calculated over the design space for starting values $x(0)$ with $x_{2}(0)=x_{4}(0)=0.999$, for $c_{p}=0.01$, $0.05,0.10,0.25,0.50,1.00$ (Fig. 5). For $c_{p}=0.01$ the spectral radius near the optimum point is less than one only in a very narrow sliver along the $x_{1}$-axis (see Fig. 5), showing that HCA will fail to converge to the true optimum point for most starting points $x(0)$. This indicates that for some values of the proportional constant, the HCA algorithm may fail to converge to the optimum point from many different starting points. HCA can also fail to converge to anything by cycling, which happens, for instance, with $x(0)=(0.999,0.999,0.999,0.999)$ and $c_{p}=1.0$.

To illustrate the convergence of the HCA method to a fixed point, the algorithm was applied for two initial designs (Fig. 6): first, starting from a fully dense initial density distribution (i.e., $x_{j}=0.999$ for $j=1,2,3,4$-for practical programming reasons, the bounds 0.001 and 0.999 are used rather than 0 and 1), and second, starting from $x_{1}=x_{3}=0.1$ and $x_{2}=x_{4}=0.999$. For both simulations, the proportional constant was set to $c_{p}=0.05$. Again, convergence was defined as no change in the objective function (7) or the design variable values $x$ larger than $10^{-7}$. For the two cases, the HCA algorithm 

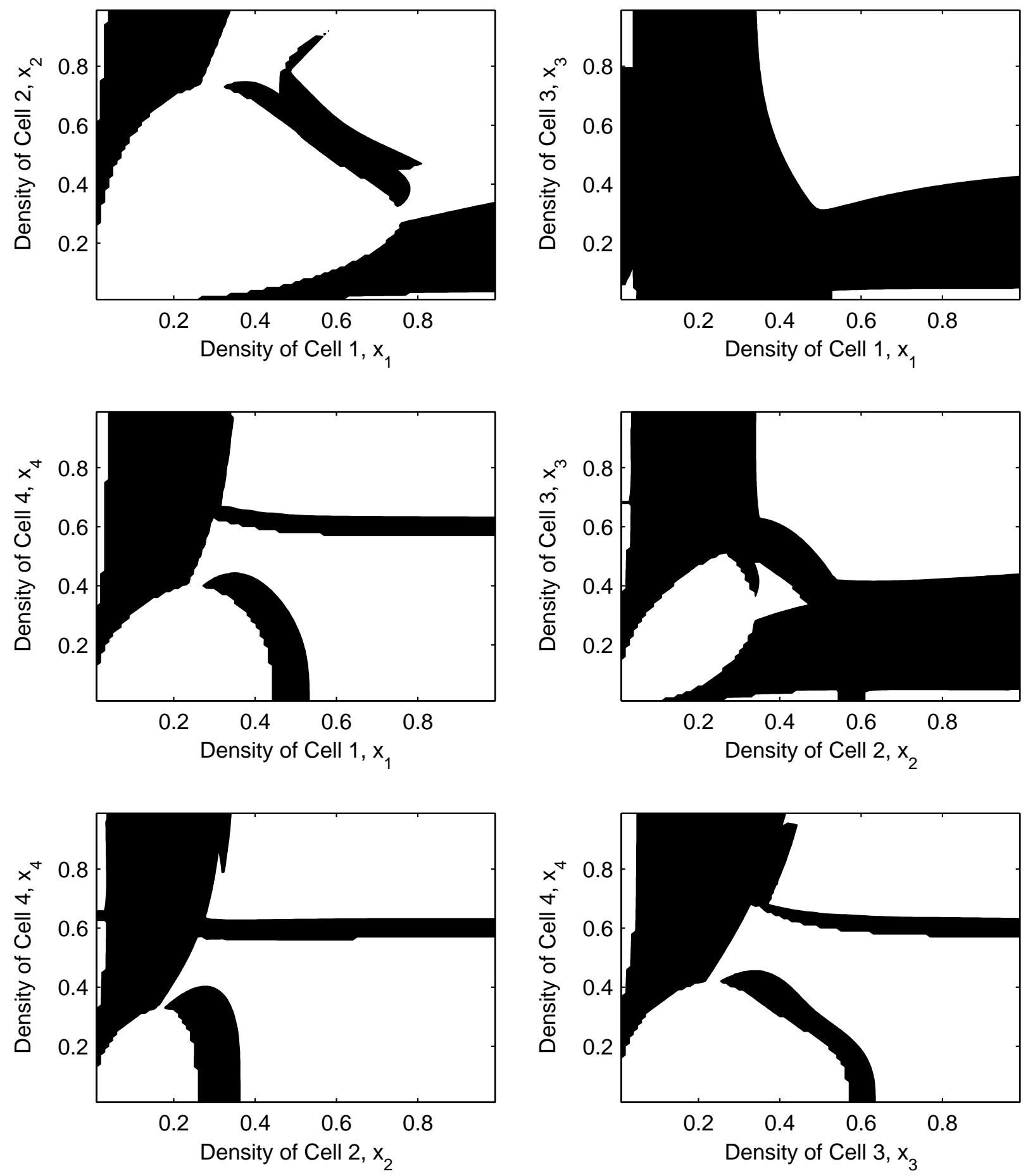

Fig. 4 Plots of spectral radius $\rho(G(x(0)))$ over the design space for combinations of two cells while the remaining two cells were set to full density, for $c_{p}=0.05 . \rho(G(x(0)))<1$ is shown in white, while $\rho(G(x(0))) \geq 1$ is shown in black.

converges to a different fixed point (see Fig. 6), neither of which is the globally optimal solution shown in Fig. 3. A summary of the convergence properties of HCA for the aforementioned initial designs, as well as several other selected designs, is recorded in Table
1. An exhaustive search of the design space was not conducted; this exercise was simply to point out that there are multiple fixed points in the design space. Each converged fixed point in Table 1 was verified with MATLAB's FMINCON as being a local 

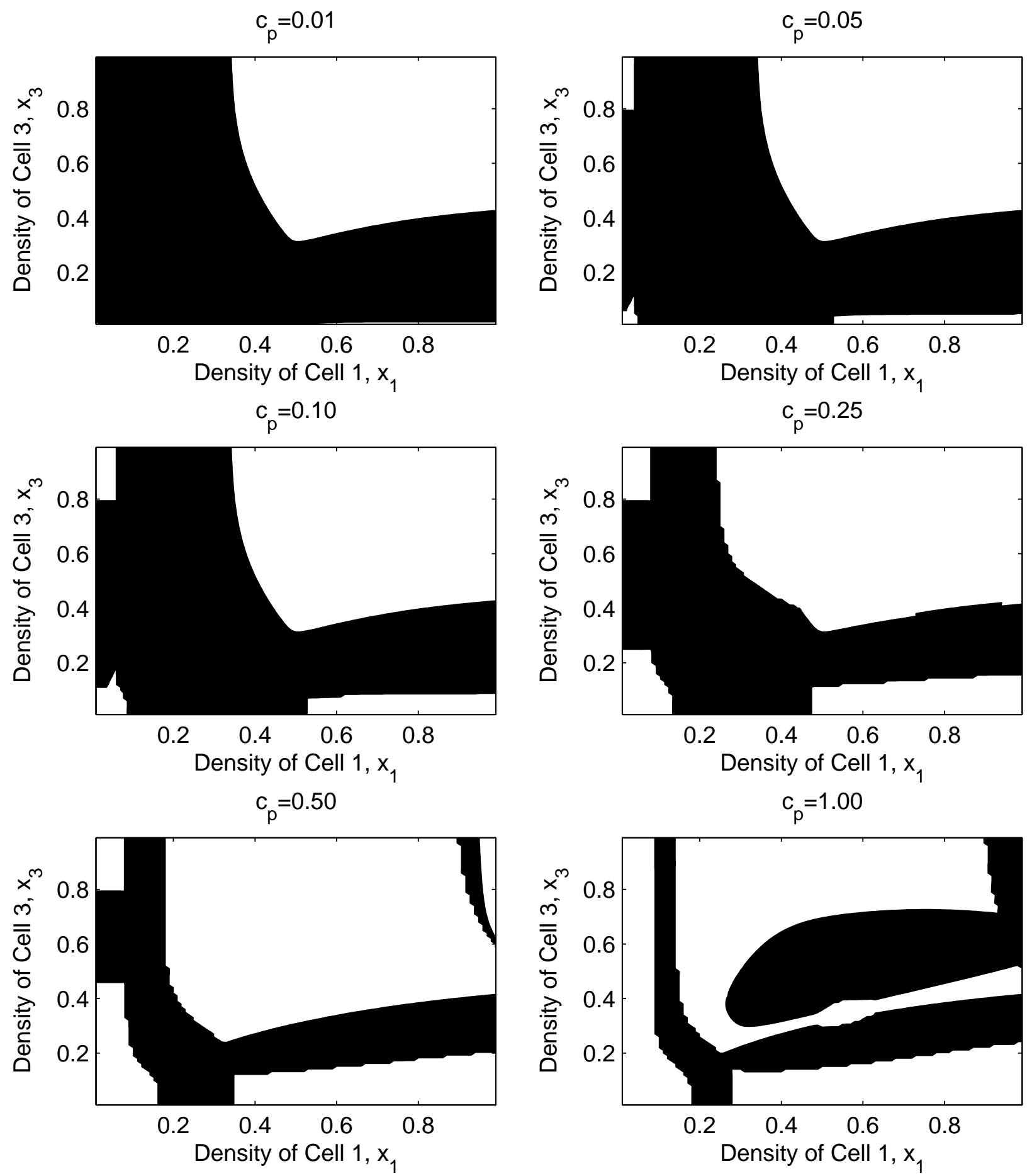

Fig. 5 Plots of spectral radius $\rho(G(x(0)))$ over the design space for starting values of Cells 1 and 3 and varying values of $c_{p}$. $\rho(G(x(0)))<1$ is shown in white, while $\rho(G(x(0))) \geq 1$ is shown in black.

minimum point.

\section{Observations from Tovar's HCA}

The HCA method presented by Tovar (2004) and Tovar et al. (2007) is a non-gradient-based optimiza- tion approach that follows mathematical principles derived from the KKT conditions. In this algorithm, the design domain is divided into cells with some communication property among neighbors. Local evolutionary rules, obtained from classical control theory, 


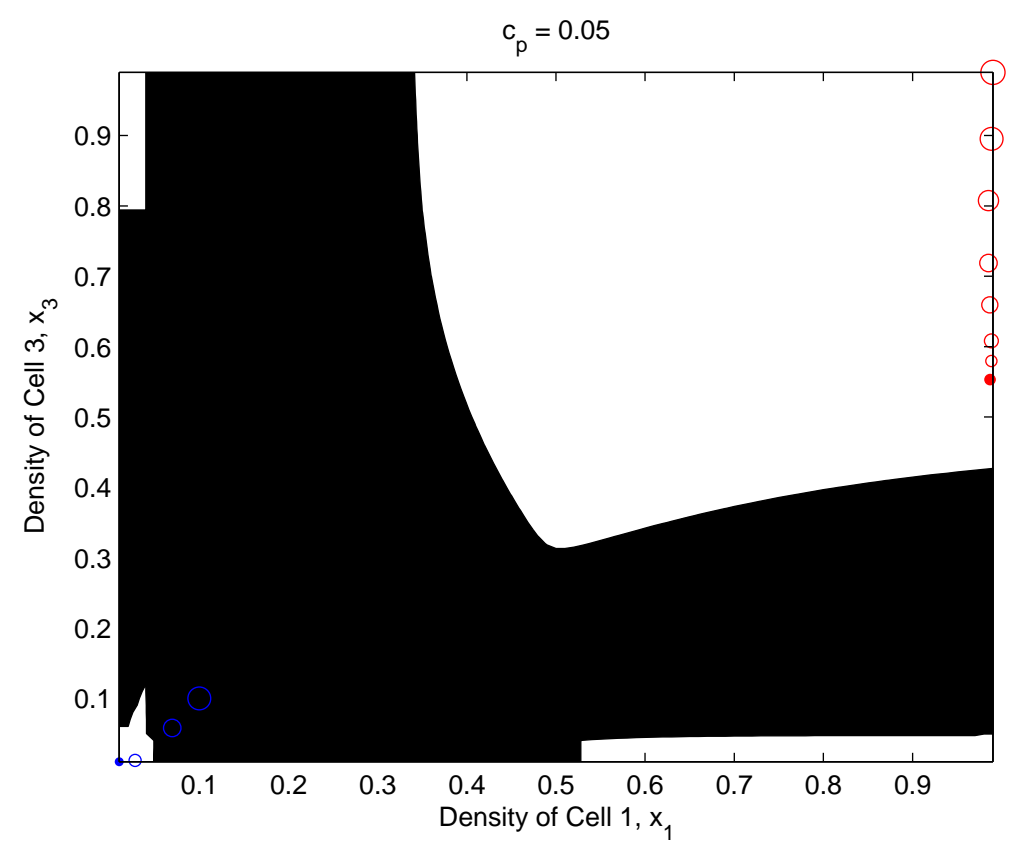

Fig. 6 Convergence history (hollow circles of decreasing radius correspond to successive iterates, while the solid circle designates the converged point) of HCA algorithm to optimal points.

\begin{tabular}{|c|c|c|}
\hline Initial point $x(0)$ & Computed fixed point & Objective function \\
\hline$(0.999,0.999,0.999,0.999)$ & $(0.987,0.999,0.553,0.999)$ & 3.79659 \\
\hline$(0.100,0.999,0.100,0.999)$ & $(0.001,0.999,0.001,0.999)$ & 4.41418 \\
\hline$(0.100,0.999,0.999,0.999)$ & $(0.987,0.999,0.553,0.999)$ & 3.79659 \\
\hline$(0.999,0.999,0.100,0.999)$ & $(0.999,0.999,0.001,0.999)$ & 3.67563 \\
\hline$(0.200,0.999,0.200,0.999)$ & $(0.999,0.999,0.001,0.999)$ & 3.67563 \\
\hline$(0.300,0.999,0.300,0.999)$ & $(0.987,0.999,0.553,0.999)$ & 3.79659 \\
\hline
\end{tabular}

Table 1 Converged HCA fixed points for $c_{p}=0.05$.

minimize the local error between a reference state $S^{*}$ and a mechanical stimulus $S$ (i.e., state variable) at each cell. These rules modify the topology of the design domain by changing the relative density $x$ of every cell (i.e., design variable). In order to avoid numerical instabilities, the algorithm defines an effective mechanical stimulus $\bar{S}$ (or effective state variable) as the average value of the cell and its neighboring cells. In Tovar et al. (2007), several examples are presented in which HCA converges to different final designs for different control parameters and neighborhood sizes. Even though it is claimed that these final designs are optimal, this is not true in a precise mathematical sense.

In engineering $\mathrm{CA}$ literature, $\mathrm{HCA} / \mathrm{CA}$ fixed points are often called "optimal points" even though such points may not be "optimal" in any precise mathematical sense. The KKT conditions used to define optimality in HCA, $S_{i}=S_{i}^{*}$, were derived for each independent cell and not for a neighborhood. The satisfaction of these optimality conditions is not guaranteed when using an averaging procedure for $S_{i}$ or $e_{i}$. Furthermore, the simple averaging procedure might result in cancellation or attenuation of the error between the mechanical stimulus and the reference state, so that $e_{i}=\bar{S}_{i}-S_{i}^{*}=0$ but $S_{i} \neq \bar{S}_{i}=S_{i}^{*}$, violating the KKT optimality conditions. Evidently, the use of the "effective state variable" induces a mathematical incorrectness that has to be addressed. For this reason, a final converged design has to be obtained for empty neighborhoods, $\hat{N}=0$.

When analyzing the convergence of the HCA algorithm, it is compulsory to change control parameters (e.g., proportional, derivative, and integral gains) and other algorithm parameters (e.g., neighborhood size), and analyze the effect on speed and the sequence of designs analyzed. However, for HCA, each control technique and neighborhood size corresponds to a different fixed point iteration function with its own fixed points. As shown in this paper, the HCA update $x(t+1)=G(x(t))$ is fixed point iteration, and $G(x(t))$ can have multiple fixed points. Depending 
on how the HCA update function $G(x(t))$ is defined, the fixed points may or may not correspond to a physical optimum point or even a mathematically local optimum point. Therefore, the convergence of HCA does not imply that a local optimum point has been found.

\section{Conclusion}

In this paper it is shown that the HCA update iteration is equivalent to fixed point iteration, a well understood mathematical process. The goal of the HCA update iteration is to converge to an optimum design, satisfying the KKT conditions. The main contribution of this paper is to prove that the HCA update iteration does in fact converge to an optimum design under the appropriate conditions. The optimality conditions for the HCA were revised and corrected in this work. Another contribution of this paper is the explicit form of the KKT conditions for the HCA update iteration.

Due to the nature of $\mathrm{HCA}$ fixed point iterative schemes, it is possible that multiple fixed points exist, of which only one or none is a physical optimum point. It has been shown for a simple example that the HCA algorithm can converge to different fixed points, depending on the initial conditions. It is important to note that a converged design found by the HCA algorithm does not imply a mathematically optimum design, unless it satisfies the KKT conditions derived in this paper. However, the results clearly show that HCA can converge to an optimal design with the appropriate initial conditions and HCA parameters.

An additional discovery, admittedly unexpected, was that the $\mathrm{HCA}$ is not globally convergent to an optimal design. This conclusion stems from evaluating the spectral radius over the design space. Disconnected regions of spectral radius less than one exist in the design space. Therefore, if the initial design does not reside within the region of spectral radius less than one (i.e., the basin of attraction) containing the optimal design, and is not sufficiently close to the optimal design, convergence of the HCA update iteration to an optimal design cannot be guaranteed. It is also possible that the HCA iteration will converge to an alternate fixed point in the design space that does not satisfy the optimality conditions.

Some past HCA studies did not achieve optimal designs as a result of an ill-posed HCA update. As previously mentioned, each control technique and neighborhood size utilized for the HCA iteration corresponds to a different fixed point iteration function with its own fixed points. Current research is being conducted on methods for achieving optimal designs with the HCA iteration using neighborhoods, because utilizing neighborhood information should reduce synthesis time as a result of the shared information between cells.

Readers interested in more convergence details are encouraged to contact the corresponding author.

\section{References}

Abdalla MM, Gürdal Z (2004) Structural design using cellular automata for eigenvalue problems. Struct Multidiscipl Optim 26:200-208

Abdalla MM, Reddy CK, Faris WF, Gürdal Z (2005) Optimal design of an electrostatically actuated micro-beam for maximum pull-in voltage. Int J Comput Struct 83:1320-1329

Bendsøe MP (1989) Optimal shape design as a material distribution problem. Struct Optim $1: 193-202$

Burks A (1970) Essays on cellular automata. In: Burks A (ed) Von Neumann's Self-reproducing Automata. University of Illinois Press, Urbana, pp 3-64

Canyurt OE, Hajela P (2005) A cellular framework for structural analysis and optimization. Comput Methods Appl Mech Eng 194:3516-3534

Chopard B, Droz M (1998) Cellular Automata Modeling of Physical Systems. Cambridge University Press, Cambridge

Currey JD (1988) The effect of porosity and mineralcontent on the Young's modulus of elasticity of compact-bone. J Biomech 21:131-139

Fyhrie DP, Carter DR (1986) A unifying principle relating stress to trabecular bone morphology. J Orthop Res 4:304-317

Gardner M (1970) The fantastic combinations of John Conway's new solitaire game "life". Sci Am 223:120-123

Gürdal Z, Tatting B (2000) Cellular automata for design of truss structures with linear and nonlinear response. In: Proceedings of the 41st AIAA/ASME/ASCE/AHS/ASC Structures, Structural Dynamics, and Materials Conference. AIAA, Atlanta

Gürdal Z, Watson LT (2001) Structural design using cellular automata. In: Proceedings of the Third International Conference on Intelligent Processing and Manufacturing of Materials. CRC Press, Boca Raton

Hajela P, Kim B (2001) On the use of energy minimization for CA based analysis in elasticity. Struct Multidiscipl Optim 23:24-33

Huiskes R, Weinans H, Grootenboer HJ, Dalstra M, Fudala B, Slooff TJ (1987) Adaptive boneremodeling theory applied to prosthetic-design analysis. J Biomech 20:1135-1150 
Huiskes R, Ruimerman R, van Lenthe GH, Janssen JD (2000) Effects of mechanical forces on maintenance and adaptation of form in trabecular bone. Nat 405:704-706

Isaacson E, Keller HB (1966) Analysis of Numerical Methods. Wiley, New York

Kita E, Toyoda T (2000) Structural design using cellular automata. Struct Multidiscipl Optim 19:64-73

Lowekamp BB, Watson LT, Cramer MS (1996) The cellular automata paradigm for the parallel solution of heat transfer problems. Parallel Algorithms Appl 9:119-130

Missoum S, Gürdal Z, Setoodeh S (2005) Study of a new local update scheme for cellular automata in structural design. Struct Multidiscipl Optim 29:103-112

Patel NM (2007) Crashworthiness design using topology optimization. Ph.D. Dissertation, Department of Aerospace and Mechanical Engineering. University of Notre Dame, Notre Dame, IN

Pesavento U (1995) An implementation of von Neumann's self-reproducing machine. Artif Life $2: 337-354$

Rozvany GIN, Zhou M (1991) Applications of COC method in layout optimization. In: Eschenauer H, Mattheck C, Olhoff N (eds) Proceedings of an International Conference on Engineering Optimization in Design Processes. SpringerVerlag, Berlin, pp 59-70

Ruimerman R, Hilbers P, van Rietbergen B, Huiskes R (2005) A theoretical framework for strain-related trabecular bone maintenance and adaptation. J Biomech 38:931-941

Ryoo J, Hajela P, Suhr J, Koratkar N (2007) Estimation of Young's modulus of single-walled carbon nanotube using cellular automata. Adv Eng Softw 38:531-537
Setoodeh S, Abdalla MM, Gürdal Z (2005) Combined topology and fiber path design of composite layers using cellular automata. Struct Multidiscipl Optim 30:413-421

Setoodeh S, Adams DB, Gürdal Z, Watson LT (2006) Pipeline implementation of cellular automata for structural design on message-passing multiprocessors. Math Comput Model 43:966-975

Setoodeh S, Gürdal Z, Watson LT (2006) Design of variable-stiffness composite layers using cellular automata. Comput Methods Appl Mech Eng $195: 836-851$

Slotta DJ, Tatting, B, Watson LT, Gürdal Z, Missoum S (2002) Convergence analysis for cellular automata applied to truss design. Eng Comput 19:953-969

Tatting B, Gürdal Z (2000) Cellular automata for design of two-dimensional continuum structures. In: Proceedings of the 8th AIAA/NASA/ISSMO Symposium on Multidisciplinary Analysis and Optimization. AIAA, Long Beach

Tovar A (2004) Bone remodeling as a hybrid cellular automaton process. Ph.D. Dissertation, Department of Aerospace and Mechanical Engineering. University of Notre Dame, Notre Dame

Tovar A, Patel NM, Niebur GL, Sen M, Renaud JE (2006) Topology optimization using a hybrid cellular automaton method with local control rules. J Mech Des 128:1205-1216

Tovar A, Patel NM, Kaushik AK, Renaud JE (2007) Optimality conditions of the hybrid cellular automata for structural optimization. AIAA J 45:673-683

Weimar JR, Tyson JJ, Watson LT (1992) Diffusion and wave propagation in cellular automaton models of excitable media. Phys D 55:309-327

Weimar JR, Tyson JJ, Watson LT (1992) Third generation cellular automaton for modeling excitable media. Phys D 55:328-339 